\title{
NONSINGULAR QUADRATIC DIFFERENTIAL EQUATIONS IN THE PLANE
}

\author{
M. I. T. CAMACHO AND C. F. B. PALMEIRA
}

\begin{abstract}
We consider the problem of determining the number of inseparable leaves of nonsingular polynomial differential equations of degree two. As a corollary of a classification theorem for the foliation defined by these equations, we prove that this number is at most 2 .
\end{abstract}

1. Introduction. The study of nonsingular differential equations in the plane is dominated by two closely related aspects: the existence of inseparable trajectories and Reeb components (since there are no closed orbits and no singularities).

Two trajectories $F_{1}$ and $F_{2}$ are said to be inseparable if for any open arcs $T_{1}$ and $T_{2}$ such that $T_{i}$ intersects transversely $F_{i}, i=1,2$, there are infinitely many trajectories that intersect both $T_{1}$ and $T_{2}$.

A Reeb component is a region of the plane where the foliation defined by the differential equation is conjugate to the foliation defined by $x d x+\left(1-x^{2}\right) d y=0$ in the region $|x| \leq 1$ (Figure 1).

For the polynomial case, L. Markus showed in [Ma] that the number of inseparable trajectories of the differential equation $P d x+Q d y=0$ is at most $6 n$ where $2 n$ is the degree of $P^{2}+Q^{2}$. Later M. P. Müller and independently S. Schecter and M. Singer [Mü, S.S.], showed this number to be at most $2 n$. Schecter and Singer obtained also examples for $n$ even, $n \geq 4$, of differential equations with $2 n-4$ inseparable trajectories.

In this paper we exhibit all possible types of topological behavior for the trajectories of the differential equation in the Poincare compactification of the plane and as a consequence we show that for $n=2$ there are at most 2 inseparable trajectories. This also answers a question raised by C. Chicone and Tian Jing Huang [C.T.] concerning the number of Reeb components that a quadratic system in the plane may admit. So we have the following.

THEOREM. Let $P$ and $Q$ be polynomials of degree at most 2 in 2 real variables, such that $P^{2}+Q^{2}$ is never zero. Consider the foliation defined by the differential equation $P d x+Q d y=0$. Then either the foliation is trivial (conjugate to the one defined by $d x=0)$ or conjugate to the one defined by $x d x+\left(1-x^{2}\right) d y=0$. We recall that a conjugacy between two foliations is a homeomorphism taking leaves of one foliation to leaves of the other foliation.

COROLLARY. There is at most one Reeb component for a nonsingular quadratic vector field in the plane.

Received by the editors July 11, 1984 and, in revised form, June 3, 1986.

1980 Mathematics Subject Classification (1985 Revision). Primary 34C35.

Both authors partially supported by $\mathrm{CNPq}-$ Brazil. 


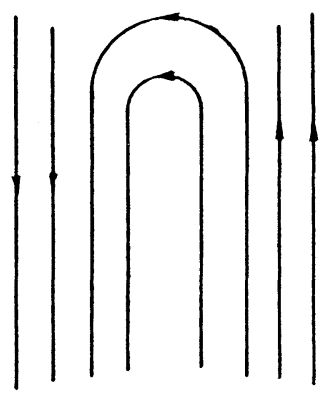

FIGURE 1

In order to prove this result we shall consider $R^{2}$ as the tangent plane to $S^{2}$ at $(0,0,1)$ and use central projection to get a foliation in the open upper hemisphere which extends (with singularities) to the equator. We will study local behavior of the foliation near the singularities and show that this local behavior determines the foliation. Computations on the sphere will be made on the coordinate system defined by central projection on the plane $x=1$. So we are actually using central projection to pass from $z=1$ to $x=1$ which amounts to the well-known change of coordinates $x=1 / Z, y=Y / Z$.

In $\S 2$ we perform this change of variables, in $\S 3$ we make the case by case analysis, leaving some technical computations to $\S 4$ (mainly blowing up singularities).

After completing this paper, the authors learned that Sheng Li-Ren $[\mathbf{S}]$ had also treated this question, with different methods. The referee informed us of the existence of another paper on this subject by A. Gasull, Sheng Li-Ren, and J. Llibre [G.S.L.].

We thank Geovan Tavares for helpful conversations and the referee for valuable suggestions.

2. Changing variables. Let us call $Y, Z$ the coordinates in the plane $x=1$. Let $h$ be the map from the $Y Z$ plane to the $x y$ plane given by $x=1 / Z, y=Y / Z$. Let $w=P(x, y) d x+Q(x, y) d y$; if $P_{i}$ and $Q_{i}$ are the homogeneous parts of $P$ and $Q$ respectively of degree $i$, then the form

$$
\begin{aligned}
Z^{4} h^{*} w & =Z\left[Q_{2}(1, Y)-Z Q_{1}(1, Y)+Z^{2} Q_{0}\right] d Y \\
& -\left[P_{2}(1, Y)+Z P_{1}(1, Y)+Z^{2} P_{0}+Y Q_{2}(1, Y)+Y Z Q_{1}(1, Y)+Y Z^{2} Q_{0}\right] d Z \\
= & Z \tilde{Q} d y-(\tilde{P}+Y \tilde{Q}) d Z
\end{aligned}
$$

extends to the whole $Y Z$ plane. We see that if $P_{2}(1, Y)+Y Q_{2}(1, Y) \not \equiv 0$ then $Z=0$ is an integral curve of $Z^{4} f^{*} w$ with singularities at the roots of $P_{2}(1, Y)+Y Q_{2}(1, Y)$. Let $V$ be the field $(\tilde{P}+Y \tilde{Q}, Z \tilde{Q})$; we have

$$
D V(Y, 0)=\left(\begin{array}{cc}
\frac{d}{d Y}\left[P_{2}(1, Y)+Y Q_{2}(1, Y)\right] & \frac{\partial}{\partial Z}(\tilde{P}+Y \tilde{Q}) \\
0 & Q_{2}(1, Y)
\end{array}\right) .
$$

Notice that whenever $P_{2}(1, Y)+Y Q_{2}(1, Y)$ is of degree less than 3 , this indicates that we have a root at $Y=\infty$. We can remove this root by a linear change of coordinates, so, there is no need to use another chart of the sphere to study all the singularities of $P d x+Q d y$ at infinity. 


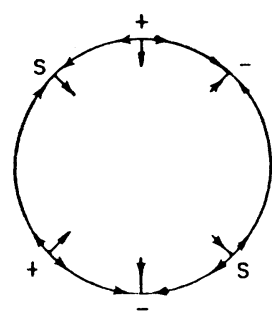

FIGURE 2

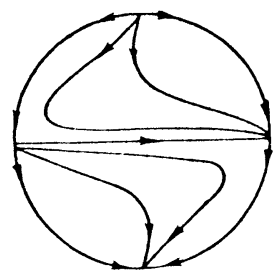

FIGURE 5

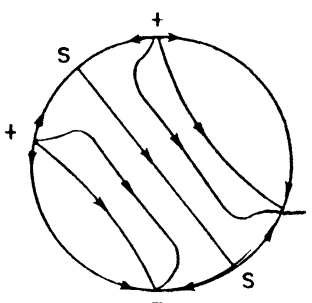

FIGURE 3

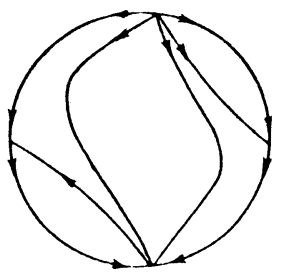

FIGURE 6

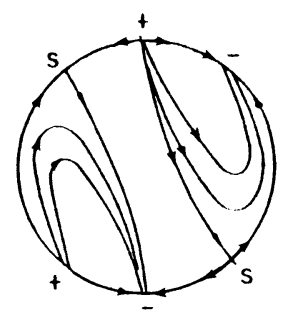

FigURE 4

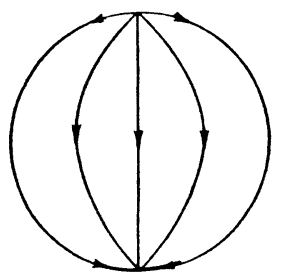

FIGURE 7

We know that to each singularity of $V$ corresponds a pair of singularities at antipodal points in the half-sphere, such that the behavior of the trajectories near a singularity for $Z>0$ is the same (except by a change of direction) as in the opposite singularity for $Z<0$, so sources correspond to sinks and saddles with unstable manifold along $Z=0$ correspond to saddles with stable manifold along $Z=0$.

If we have 6 hyperbolic singularities in 3 pairs as above, then the only possibility is the one of Figure 2, where we denote the sources by + , the sinks by -, and the saddles by $S$. So the only possible foliations are the ones of Figures 3 and 4 .

Actually, all we need is that one of the diagonal terms of $D V\left(Y_{0}, 0\right)$ is nonzero. We know then that the singularity is either a node, a saddle, or a saddle-node $[\mathbf{T}]$, and this is sufficient to determine the foliation in the half-sphere.

3. The case by case analysis. (a) $P_{2}$ and $Q_{2}$ are relatively prime. Then $P_{2}(1, Y)+Y Q_{2}(1, Y)$ and $Q_{2}(1, Y)$ are relatively prime also, so all singularities have hyperbolicity in the $Z$ direction.

(a1) If $P_{2}(1, Y)+Y Q_{2}(1, Y)$ has 3 simple roots (all real), then all singularities are hyperbolic, and, as we said before, we have either Figure 3 or Figure 4. Figure 3 can be realized by $2 x y d x-\left(x^{2}+y^{2}+1\right) d y=0$ and Figure 4 by $y^{2} d x+\left(x^{2}+1\right) d y=0$.

(a2) If $P_{2}(1, Y)+Y Q_{2}(1, Y)$ has one simple and one double root, then we have a pair of antipodal hyperbolic singularities and another antipodal pair that has hyperbolicity only along the $Z$-direction. Considering the vector field restricted to the boundary of the half-sphere, it becomes clear that we have 2 saddle-nodes at the points corresponding to the double root, and a source-sink pair corresponding to the simple root. The foliation, then, is either the one of Figure 5 or the one of Figure 6. Figure 5 can be realized by $\left(x y+y^{2}\right) d x-\left(x^{2}+1\right) d y=0$ and Figure 6 by $\left(x y+y^{2}+1\right) d x-x^{2} d y=0$.

(a3) If $P_{2}+Y Q_{2}$ has a triple root, we have a single pair of singularities and the hyperbolicity in the $Z$-direction imposes that it must be a source-sink pair. We 


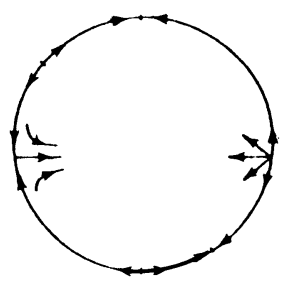

FiguRE 8

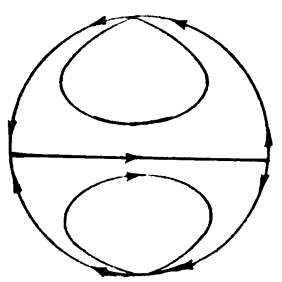

FIGURE 10

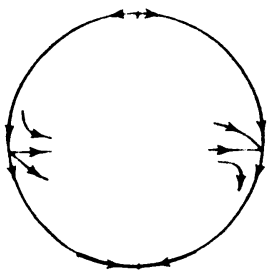

Figure 9

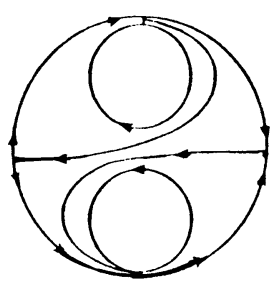

FIGURE 11

have Figure 7. This case can be realized by $x y d x-\left(x^{2}+y^{2}+1\right) d y=0$.

(a4) If $P_{2}+Y Q_{2}$ has a pair of complex roots, we have only one pair of singularities, and they are hyperbolic, so again we have Figure 7 . This case can be realized by $\left(y^{2}+1\right) d x+\left(x^{2}-x y+y^{2}\right) d y=0$.

(b) $P_{2}$ and $Q_{2}$ have one (and only one) common factor:

$$
P_{2}(x, y)=(a x+b y)(c x+e y) \quad \text { and } \quad Q_{2}(x, y)=(a x+b y)\left(c^{\prime} x+e^{\prime} y\right) .
$$

(b1) If $P_{2}(1, Y)+Y Q_{2}(1, Y)$ has 3 simple (real) roots we have two pairs of hyperbolic singularities and one pair of singularities with hyperbolicity along the $Y$ direction only (this corresponds to the common root of $P_{2}(1, Y)$ and $P_{2}(1, Y)+$ $\left.Y Q_{2}(1, Y)\right)$. If the semihyperbolic singularities form a pair of saddle-nodes we would have the case of Figure 8 which cannot be completed to a nonsingular vector field, so it is either a saddle-saddle pair or a source-sink pair and what we get is again Figure 3 or 4 (remember that the other antipodal pairs of singularities are either sink-source or saddle-saddle). Figure 3 can be realized by $-3 y(x-y) d x+$ $\left(x^{2}-y^{2}+1\right) d y=0$ and Figure 4 by $y(x-y) d x+\left(x^{2}-y^{2}+1\right) d y=0$.

(b2) If $P_{2}(1, Y)+Y Q_{2}(1, Y)$ has a double root we have two different situations.

(b2.1) The double root is not the common root of $P_{2}$ and $Q_{2}$. We have two pairs of singularities, one hyperbolic only in the $Z$-direction and the other hyperbolic only in the $Y$-direction. The double root generates a pair of saddle-nodes, so we have Figure 9 and the only possible ways of completing the picture are the ones of Figures 5 and 6 . Figure 5 can be realized by $x y d x-[(x+y) x+1] d y=0$ and Figure 6 by $(x y-1) d x-x(x+y) d y=0$.

(b2.2) The double root is the common root of $P_{2}$ and $Q_{2}$. We have a pair of hyperbolic singularities (corresponding to the simple root) and a pair of singularities where the linear part of the vector field is $\left(\begin{array}{ll}0 & * \\ 0 & 0\end{array}\right)$. Blowing up this singularity we see that we have either Figure 7 or 10 or 11 . Figure 7 can be realized by $\left(y^{2}+1\right) d x$ $+x y d y=0$, Figure 10 by $\left(y^{2}+1\right) d x-2 x y d y=0$, Figure 11 by $\left(y^{2}+1\right) d x-$ $(2 x y+1) d y=0$. 


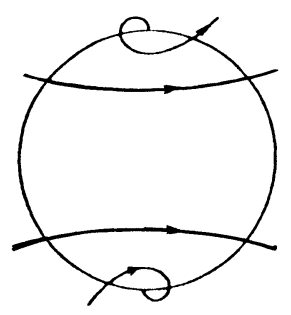

FIGURE 12

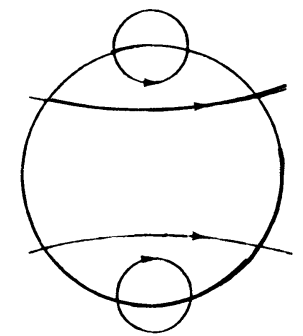

FIGURE 13

(b3) $P_{2}(1, Y)+Y Q_{2}(1, Y)$ has a triple root. There is only one singularity, which we blow up to get Figure 7. This case can be realized by $\left(y^{2}+1\right) d x+\left(y^{2}-x y\right) d y=0$.

(b4) $P_{2}+Y Q_{2}$ has 2 complex roots. It is easy to see that we have only one pair of singularities with hyperbolicity along the $Y$-direction. Just as in case (a4) we have Figure 7. This case can be realized by $x y d x+\left(y^{2}+1\right) d y=0$.

(b5) $P_{2}(1, Y)+Y Q_{2}(1, Y) \equiv 0$. This happens if $c=e^{\prime}=0$ and $c^{\prime}=-e$; then $P_{2}(x, y)=(a x+b y) y$ and $Q_{2}(x, y)=-(a x+b y) x$. In this case, we see that $V$ becomes

$V=\left(Z\left(P_{1}(1, Y)+Y Q_{1}(1, Y)\right)+Z^{2}\left(P_{0}+Y Q_{0}\right) ; Z Q_{2}(1, Y)+Z^{2} Q_{1}(1, Y)+Z^{3} Q_{0}\right)$.

We consider $\bar{V}=V / Z$ which extends to $Z=0$ as $\left(P_{1}+Y Q_{1}, Q_{2}\right)$. We will study $\bar{V}$ in $\S 4$ and see that we get the situation of Figures 12 and 13 . Figure 12 can be realized by $\left(y^{2}+1\right) d x-(x y-1) d y=0$ and Figure 13 by $\left(y^{2}+y+1\right) d x-(x y-1) d y=0$.

(c) $P_{2}$ and $Q_{2}$ have two common factors, i.e., $P_{2}=m Q_{2}$. If $m \neq 0$ we change variables: $x=u, y=-m u+v$ getting an equation with $m=0$ :

$$
[a u+b v+c] d u+\left[e u^{2}+f u v+g v^{2}+h u+i v+j\right] d v=0 .
$$

(c1) $Y Q_{2}(1, Y)$ has 3 simple (real) roots (remember $P_{2} \equiv 0$ ). Then we have 3 pairs of singularities, all hyperbolic in the $Y$-direction and one $(Y=0, Z=0)$ also hyperbolic in the $Z$-direction, so one pair of antipodal singularities is a source-sink pair (since $\left.\left.(d / d Y)\left(Y Q_{2}(1, Y)\right)\right|_{Y=0}=Q_{2}(1,0)\right)$ and the other two pairs can be source-sink, saddle-saddle or saddle-node saddle-node. Examining all possibilities we see that we can have Figures $3,4,14$, and 15 .

Figure 3 can be realized by $d x+\left(x^{2}-y^{2}+1\right) d y=0$ and Figure 4 by $d x+$ $\left(x^{2}+x y\right) d y=0$.

Figure 14 can be realized by $y d x+\left(x^{2}-y^{2}+1\right) d y=0$ and Figure 15 by $(-2 x+y) d x+\left(x^{2}-y^{2}-1\right) d y=0$.

(c2) $Y Q_{2}(1, Y)$ has one double root and one simple root.

(c2.1) The double root is zero. We have a singularity hyperbolic in the $Y$ direction only, $\left(Y_{0}, 0\right)$, and another one, $(0,0)$, with $D V(0,0)=\left(\begin{array}{ll}0 & * \\ 0 & 0\end{array}\right)$. Blowing up this singularity we see that we have either Figure 16 or Figure 7 or Figure 10 or Figure 11. Figure 16 can be realized by $(x-1) d x+\left(x y+y^{2}+x-y\right) d y=0$. Figure 7 can be realized by $d x+\left(x y+y^{2}\right) d y=0$. Figure 10 can be realized by $-d x+x y d y=0$. Figure 11 can be realized by $-d x+(x y+y) d y=0$.

(c2.2) The double root is not zero. The equation is then $(a x+b y+c) d x+$ $\left[(e x+f y)^{2}+m x+n y+p\right] d y=0$ with $e \neq 0$. Changing variables again $(u=e x+f y$, $v=y)$ the equation to consider is $(a x+b y+c) d x+\left(x^{2}+n y+p\right) d y=0$. If $n=0$ 


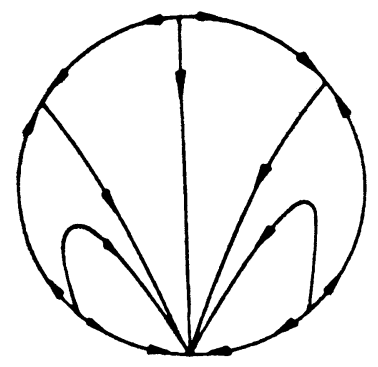

FIGURE 14

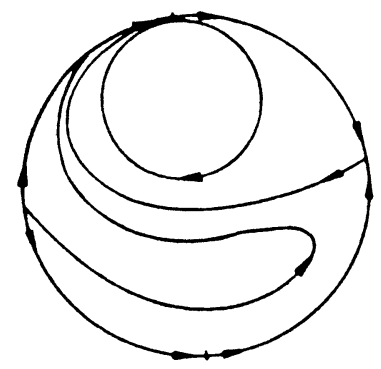

FIGURE 16

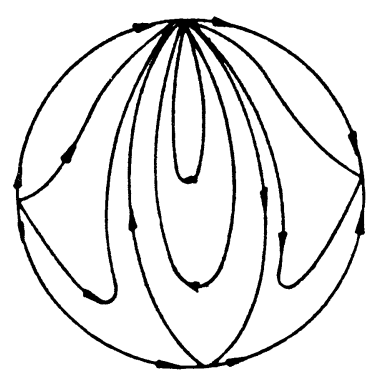

FIGURE 18

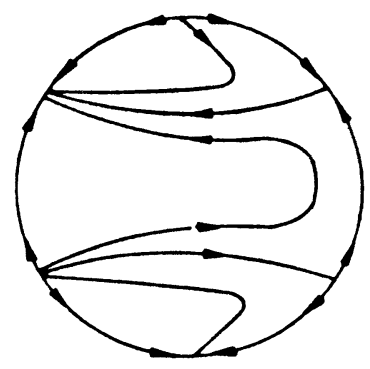

FIGURE 15

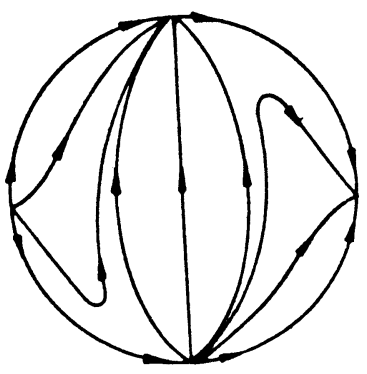

FIGURE 17

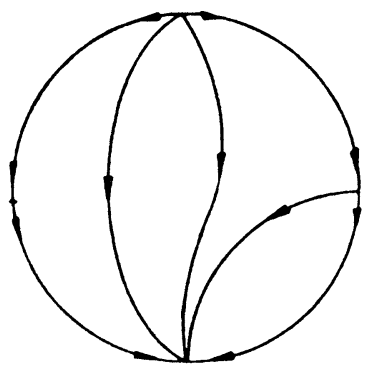

FIGURE 19

and $p<0$, then $b=0$, and we have $(a x+c) d x+\left(x^{2}-q^{2}\right) d y=0$. Integrating this equation it is easy to see that if $|c / a|>|q|$ we have a trivial foliation (this includes $a=0$ ) and if $|c / a|<|q|$ we have a foliation with a Reeb component. The corresponding figures in the half-sphere are Figures 17 and 18.

If $n \neq 0$ or if $n=0$ and $p>0$ we see that $P_{2}+Y Q_{2}=Y$; so the double root is at infinity. We will simply switch $x$ and $y$ and work with the equation $\left(y^{2}+n x+p\right) d x+(a y+b x+c) d y=0$. Now $V$ has a degenerate singularity at $(0,0)$ which we will blow up, getting Figure 19 if $n \neq 0$ and Figure 7 if $n=0, p>0$. Figure 17 can be realized by $(x-2) d x+\left(x^{2}-1\right) d y=0$. Figure 18 can be realized by $x d x+\left(x^{2}-1\right) d y=0$. Figure 19 can be realized by $(y-1) d x+\left(x^{2}+y\right) d y=0$.

(c3) $Y Q_{2}(1, Y)$ has a triple root. Our equation can be written, then, as $(a x+b y+c) d x+\left(y^{2}+e x\right) d y=0$ and, if $a \neq 0$, blowing up the origin for the vector field $V$, we see that we have again Figure 7 . If $a=0$ we can easily integrate the 
equation and see that we get Figure 17 if $b \neq 0$ and Figure 7 if $b=0$.

(c4) $Y Q_{2}(1, Y)$ has a pair of complex roots. We have only one singularity, which is hyperbolic in the $Y$-direction, and as before we have Figure 7 . This case can be realized by $(x+1) d x+\left(x^{2}+y^{2}\right) d y=0$.

This concludes the case by case analysis. Notice that except in cases (c1) and (c2.2), there is always a transversal arc that intersects once every leaf on the open hemisphere. This implies that the foliation is trivial (trivialization lemma in $[\mathbf{P}]$ ).

4. Blowing up summary. Blowing up is a well-known method for studying isolated degenerate singularities of vector fields. In order to fix notations we will briefly present the basic facts which will be used in this paper. The interested reader can consult $[\mathbf{S . S . 2}]$ or $[\mathbf{A}]$ for more details.

Let $V(Y, Z)=\left(V_{1}, V_{2}\right)$ be an analytic vector field in $R^{2}$, with $V_{1}=V_{1}^{d}+V_{1}^{d+1}+$ $\cdots$ and $V_{2}=V_{2}^{d}+V_{2}^{d+1}+\cdots$ where $V_{i}^{j}$ is a homogeneous polynomial of degree $j$, $d \geq 1$, and $\left(V_{1}^{d}\right)^{2}+\left(V_{2}^{d}\right)^{2} \neq 0$.

Let $f: R^{2} \rightarrow R^{2},(y, z) \mapsto(Y, Z)$ be defined by $f(y, z)=(y, y z)$. The image of $f$ is the plane with the set $\{(0, Z), Z \neq 0\}$ removed. $f$ collapses the $z$-axis to $(0,0)$ and takes each line $z=z_{0}$ to the line $Z=z_{0} Y$. The half-plane $y>0$ (respectively $y<0$ ) is taken diffeomorphically onto the half-plane $Y>0$ (respectively $Y<0$ ).

Pull back $V \mid R^{2}-\{Z$-axis $\}$ by $f$ to obtain a vector field $U=f^{*} V$ on $R^{2}-\{z$-axis $\}$. $U=\left(U_{1}, U_{2}\right)$ is defined by

$$
\begin{aligned}
U_{1}(y, z) & =V_{1}(y, y z)=y^{d} V_{1}^{d}(1, z)+y^{d+1} V_{1}^{d+1}(1, z)+\cdots \\
U_{2}(y, z) & =\frac{1}{y}\left[V_{2}(y, y z)-z V_{1}(y, y z)\right] \\
& =y^{d-1}\left(V_{2}^{d}(1, z)-z V_{1}^{d}(1, z)\right)+y^{d}\left(V_{2}^{d+1}(1, z)-z V_{1}^{d+1}(1, z)\right)+\cdots .
\end{aligned}
$$

Since $d \geq 1, U$ extends to an analytic vector field, in the whole plane, which we still call $U$. There are 2 cases to consider:

(1) $Y V_{2}^{d}(Y, Z)-Z V_{1}^{d}(Y, Z) \not \equiv 0$. We may divide $U$ by $y^{d-1}$ to get a new analytic vector field $U^{\prime} . U$ and $U^{\prime}$ have the same solution curves on $R^{2}-\{z$-axis $\}$, so $f$ sends trajectories of $U^{\prime}$ to trajectories of $V$. The $z$-axis is invariant under $U^{\prime}$ and there are at most $d+1$ singularities of $U^{\prime}$ on the $z$-axis. If any of these singularities is degenerate we repeat the procedure.

(2) $Y V_{2}^{d}(Y, Z)-Z V_{1}^{d}(Y, Z) \equiv 0$. This case will not occur in this paper.

Clearly $U^{\prime}$ gives a complete picture of the solution curves of $V$ in a neighborhood of $(0,0)$ with the $Z$-axis removed. To complete the picture we use the map $g(y, z)=$ $(y z, z)$. The image of $g$ is the plane with $\{(Y, 0) ; Y \neq 0\}$ removed. $g$ collapses the $y$-axis to $(0,0)$ and takes lines $y=y_{0}$ into lines $Y=y_{0} Z$. Pull back $V \mid R^{2}$ $\{Y$-axis $\}$ by $g$ to obtain a vector field $S=g^{*} V$ on $R^{2}-\{y$-axis $\}$, which can be extended to an analytic vector field in $R^{2}$. $S=\left(S_{1}, S_{2}\right)$ is given by $S_{1}(y, z)=$ $z^{-1}\left[V_{1}(y z, z)-y V_{2}(y z, z)\right]$ and $S_{2}(y, z)=V_{2}(y z, z)$.

As before, if $Y V_{2}^{d}-Z V_{1}^{d} \not \equiv 0$ (and this is the case in this paper), we can divide by $z^{d-1}$ and get a new analytic vector field $S^{\prime}$ which leaves the $y$-axis invariant and has at most $d+1$ singularities, on the $y$-axis. As we are interested only in the trajectories of $S^{\prime}$ near the $z$-axis (to complete our picture of the trajectories of $V$ ), we have to consider only two cases:

(i) $S^{\prime}$ is nonsingular at $(0,0)$ and by the invariance of the $y$-axis, it is almost horizontal near $(0,0)$. 

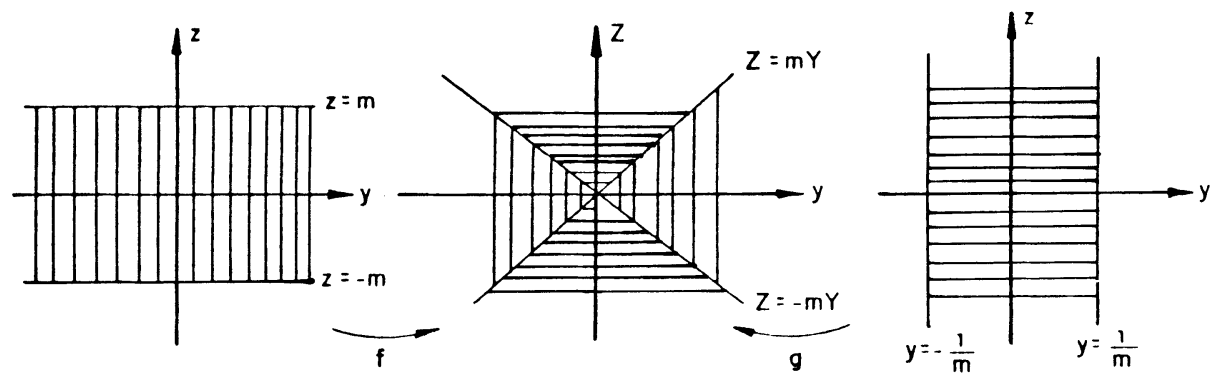

FIGURE a

(ii) $S^{\prime}(0,0)=0$, and we will study this singularity, blowing up again, if necessary (in this paper we will only encounter here hyperbolic singularities).

4.1. (b2.2) and (b.3) cases. Our equation is

$[(a x+b y)(c x+e y)+m x+n y+p] d x+\left[(a x+b y)\left(c^{\prime} x+e^{\prime} y\right)+m^{\prime} x+n^{\prime} y+p^{\prime}\right] d y=0$

with the condition that $P=0$ and $Q=0$ do not intersect and $-a / b$ is a simple root of $c+\left(e+c^{\prime}\right) Y+e^{\prime} Y^{2}$. By a simple change of coordinates, it is sufficient to consider $a=0$ and $b=1$. Then we must have $c=m=0, e \neq 0, c^{\prime} \neq 0, e+c^{\prime} \neq 0$, and $n^{2}-4 e p<0$ (nonintersection condition).

We have

$$
\begin{gathered}
\left(e y^{2}+n y+p\right) d x+\left[y\left(c^{\prime} x+e^{\prime} y\right)+m^{\prime} x+n^{\prime} y+p^{\prime}\right] d y=0, \\
V=\left(V_{1}, V_{2}\right) \\
=\left(e Y^{2}+n Y Z+p Z^{2}+Y\left(c^{\prime} Y+e^{\prime} Y^{2}+m^{\prime} Z+n^{\prime} Y Z+p^{\prime} Z^{2}\right) ;\right. \\
\left.Z\left(c^{\prime} Y+e^{\prime} Y^{2}+m^{\prime} Z+n^{\prime} Y Z+p^{\prime} Z^{2}\right)\right) .
\end{gathered}
$$

The singularities of $V$ are $(0,0)$ and $\left(\left(e+c^{\prime}\right) /\left(-e^{\prime}\right), 0\right)$

$$
D V(Y, 0)=\left(\begin{array}{cc}
2\left(e+c^{\prime}\right) Y+3 e^{\prime} Y^{2} & * \\
0 & c^{\prime} Y+e^{\prime} Y^{2}
\end{array}\right)=\left(\begin{array}{cc}
\lambda_{1} & * \\
0 & \lambda_{2}
\end{array}\right)
$$

if $Y=\left(e+c^{\prime}\right) /\left(-e^{\prime}\right)$ we have $\lambda_{1}=\left(e+c^{\prime}\right)^{2} / e^{\prime}$ and $\lambda_{2}=\left(e+c^{\prime}\right) e / e^{\prime}$. So we have a source-sink pair if $\left(e+c^{\prime}\right) e>0$ and a saddle-saddle pair if $\left(e+c^{\prime}\right) e<0$. Remember that if $e+c^{\prime}=0$ we have a triple root and this is (b.3); and if $e=0$, $n^{2}-4 p e=n^{2} \geq 0$.

Blowing up $(0,0)$ we get $U=\left(U_{1}, U_{2}\right)=y U^{\prime}$ given by

$$
\begin{aligned}
U_{1} & =\left(e+c^{\prime}\right) y^{2}+\left(n+m^{\prime}\right) y^{2} z+p y^{2} z^{2}+e^{\prime} y^{3}+n^{\prime} y^{3} z+p^{\prime} y^{3} z^{2} \\
& =y\left[\left(e+c^{\prime}\right) y+\cdots\right], \\
U_{2} & =-e y z-n y z^{2}-p y z^{3}=y[-e x+\cdots] .
\end{aligned}
$$

It is clear that $(0,0)$ is the only singularity of $U^{\prime}$ and

$$
D U^{\prime}(0,0)=\left(\begin{array}{cc}
e+c^{\prime} & 0 \\
0 & -e
\end{array}\right) \text {. }
$$

Since $g^{*} V=z S^{\prime}$ with $S^{\prime}(0,0) \neq 0$ we have Figure a if $\left(e+c^{\prime}\right) e>0$ and Figure b if $\left(e+c^{\prime}\right) e<0$. 


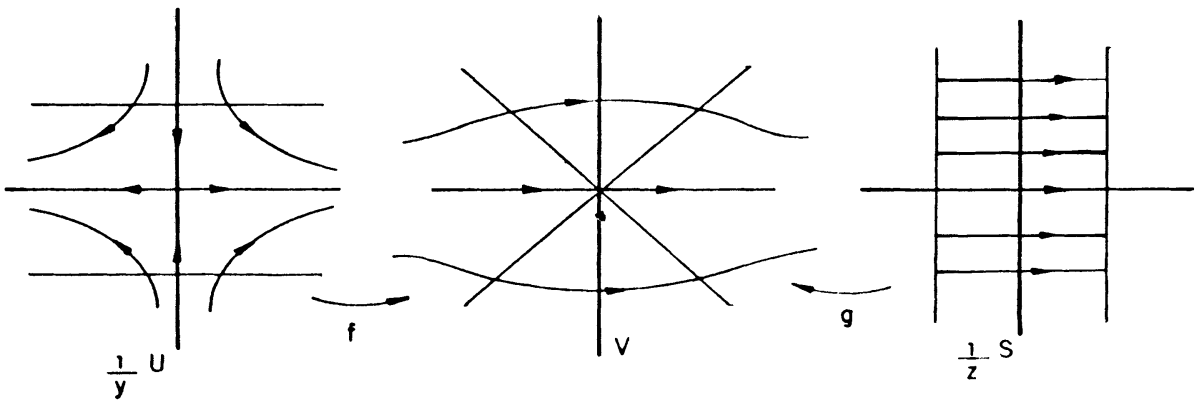

FIGURE b
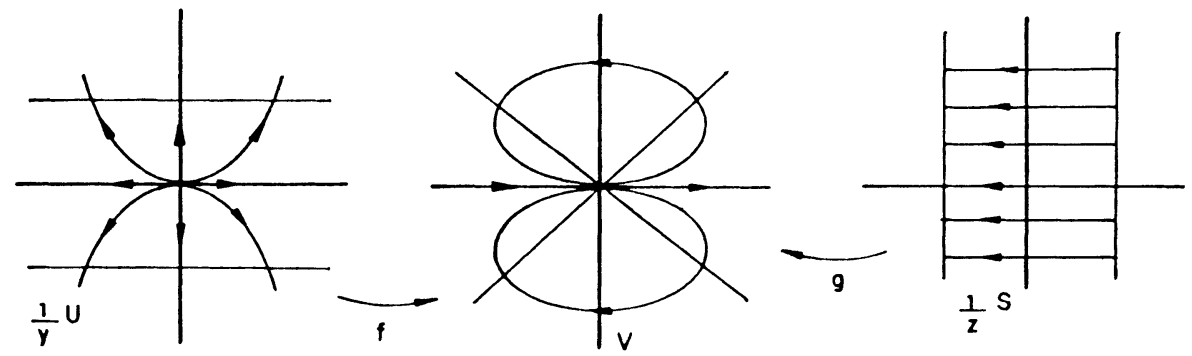

FIGURE $\mathrm{c}$ 11.

It is clear that Figure a leads to Figure 7 and Figure b leads to Figures 10 and

If $e+c^{\prime}=0$ (case (b.3)), $U^{\prime}$ has a semihyperbolic singularity at $(0,0)$ and since $U^{\prime}(y, 0)=\left(e^{\prime} y^{2}, 0\right)$ we get Figure $\mathrm{c}$ which leads to Figure 7.

4.2. The (c2.1) case. As before, we simplify the equation before blowing up: Since $Y=0$ is a double root of $Y Q_{2}(1, Y)$, in the equation

$$
(a x+b y+c) d x+\left(e x^{2}+f x y+g y^{2}+h x+i y+j\right) d y=0
$$

we must have $e=0 \neq f$.

A simple change of coordinates will transform this into an equation with $f=1$, $j=0$, so we need only to consider $(a x+b y+c) d x+\left(x y+g y^{2}+h x+i y\right) d y=0$. We have

$$
\begin{aligned}
& V_{1}=a Z+(b+h) Y Z+c Z^{2}+Y^{2}+g Y^{3}+i Y^{2} Z \\
& V_{2}=Y Z+g Y^{2} Z+h Z^{2}+i Y Z^{2} .
\end{aligned}
$$

Blowing up $(0,0)$ by the map $f$, we get $U=\left(U_{1}, U_{2}\right)$ given by

$$
\begin{aligned}
& U_{1}=a y z+y^{2}+(b+h) y^{2} z+c y^{2} z^{2}+g y^{3}+i y^{3} z \\
& U_{2}=-a z^{2}-b y z^{2}-c y z^{3} .
\end{aligned}
$$

We have two subcases according to whether $a \neq 0$ or $a=0$.

1. $a \neq 0$. $U(0, z)=\left(0,-a z^{2}\right)$ so the only singularity of $U$ is $(0,0)$ and since $D U(0,0)=0$ we must blow up $(0,0)$ for the vector field $U$. Let $f^{*} U=\bar{U}$ and $g^{*} U=\tilde{U}$. We have

$$
\begin{aligned}
& \bar{U}_{1}=a y^{2} z+y^{2}+(b+h) y^{3} z+c y^{4} z^{2}+g y^{3}+i y^{4} z \\
& \bar{U}_{2}=-2 a y z^{2}-y z-(2 b+h) y^{2} z^{2}-2 c y^{3} z^{3}-g y^{2} z-i y^{3} z^{2} .
\end{aligned}
$$



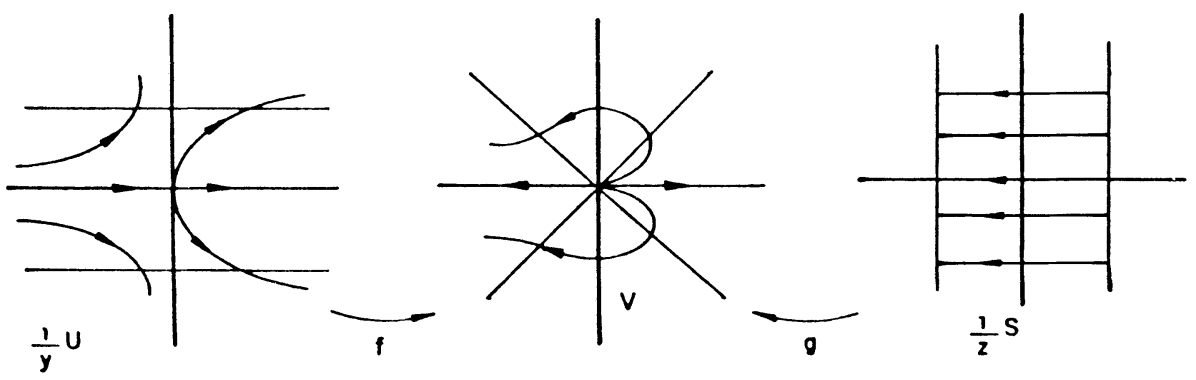

Figure d
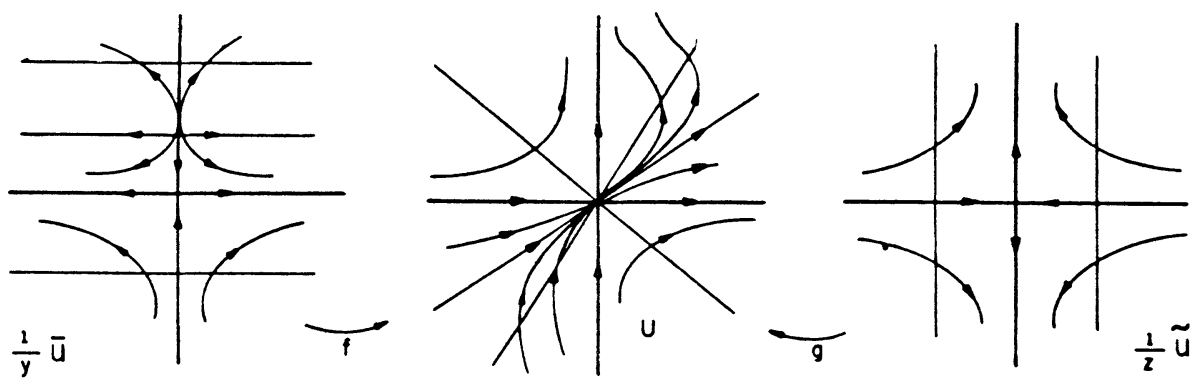

Figure e

$\bar{U} / y$ has singularities $(0,0)$ and $(0,-1 / 2 a)$. Its derivative at $(0,0)$ is $\left(\begin{array}{cc}1 & 0 \\ 0 & -1\end{array}\right)$ and at $(0,-1 / 2 a)$ is $\left(\begin{array}{rr}1 / 2 & 0 \\ * & 1\end{array}\right)$.

$$
\begin{aligned}
& \tilde{U}_{1}=2 a y z+y^{2} z+(2 b+h) y^{2} z^{2}+2 c y^{2} z^{3}+i y^{3} z^{3} \\
& \tilde{U}_{2}=-a z^{2}-b y z^{3}-c y^{2} z^{4} .
\end{aligned}
$$

$\tilde{U} / z$ has singularities $(0,0)$ and $(-2 a, 0)$ with derivatives respectively $\left(\begin{array}{cc}2 a & 0 \\ 0 & -a\end{array}\right)$ and $\left(\begin{array}{cc}-2 a & * \\ 0 & -a\end{array}\right)$.

Then we have the situation of Figure d.

Since $g^{*} V$ is nonsingular at $(0,0), V$ is given by Figure e.

REMARK. Figures d and e were drawn for $a<0$. If $a>0$, the pictures would be analogous. It is easy to see that Figure e leads to Figure 16.

2. $a=0$. Looking at the equation $(b y+c) d x+\left(x y+g y^{2}+h x+i y\right) d y=0$ it is clear that it is nonsingular only if $c \neq 0$ and either $b=0$ or if $b \neq 0, h=c / b$ and $g c / b \neq i$. Dividing $U$ by $y$ we get

$$
U_{1} / y=y+(b+h) y z+c y z^{2}+i y^{2} z ; \quad U_{2} / y=-b z^{2}-c z^{3} .
$$

2.1. $b \neq 0$. $U / y$ has singularities at $(0,0)$ and $(0,-b / c)$ with derivatives

$$
\left(\begin{array}{ll}
1 & 0 \\
0 & 0
\end{array}\right) \text { and }\left(\begin{array}{cc}
0 & 0 \\
0 & -b^{2} / c
\end{array}\right)
$$

respectively, and both points are saddle-nodes.

2.2. $b=0 . U / y$ has $(0,0)$ as its only singularity, with derivative $\left(\begin{array}{ll}1 & 0 \\ 0 & 0\end{array}\right)$, and it is a source if $c<0$ and a saddle if $c>0$. 

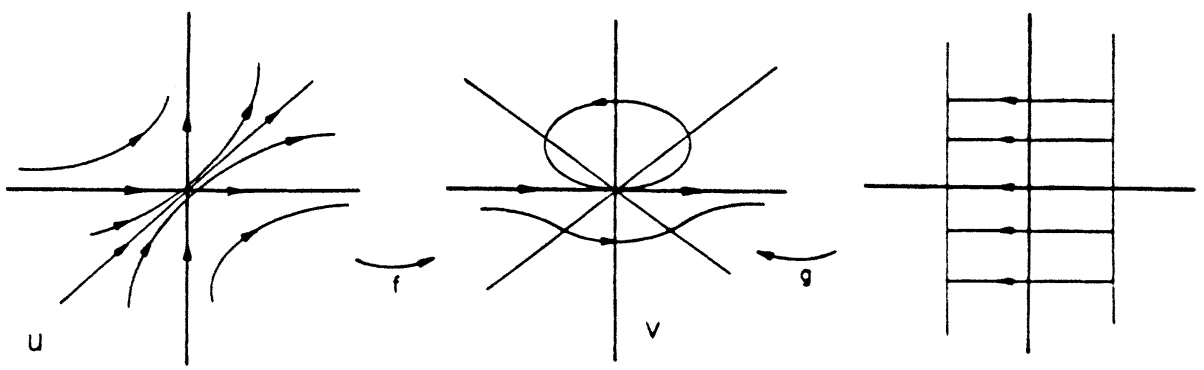

Figure $\mathrm{f}$
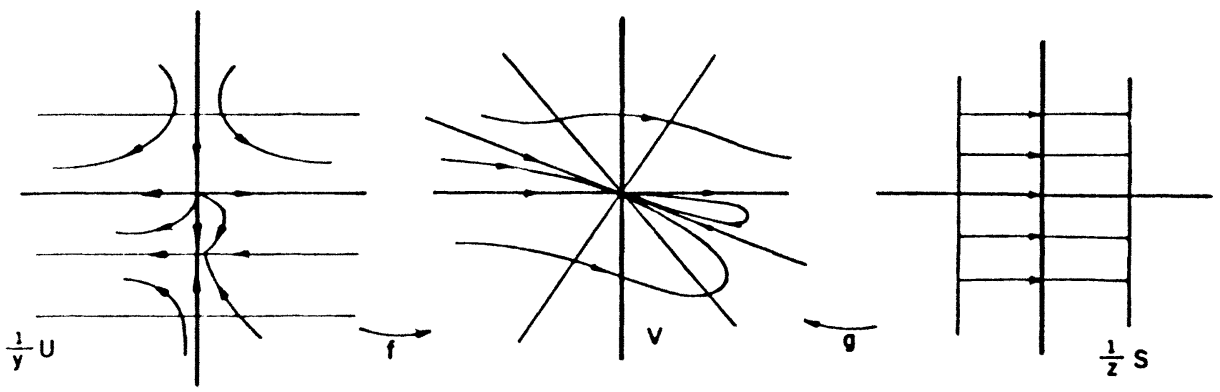

FIGURE g
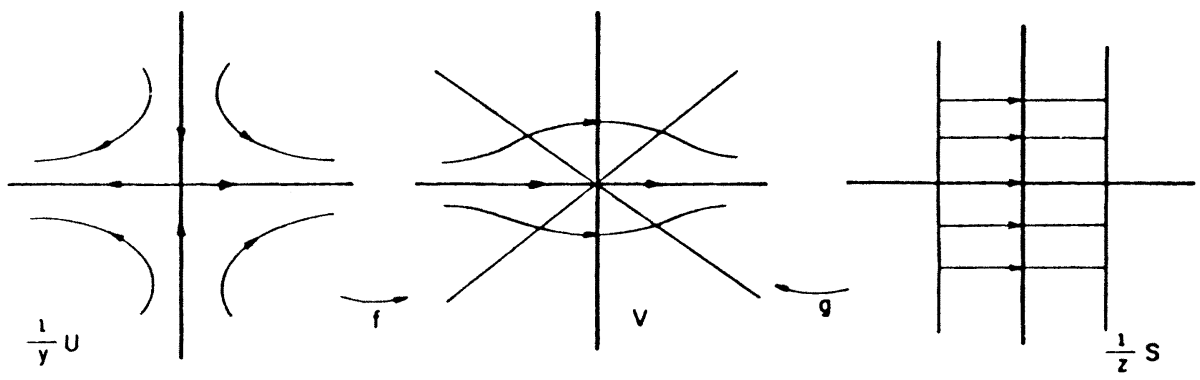

FIGURE h

Since $g^{*} V=z S^{\prime}$ and $S^{\prime}$ is nonsingular at $(0,0)$, we have Figure $\mathrm{f}$ if $b \neq 0$, Figure $\mathrm{g}$ if $b=0$ and $c>0$, and Figure $\mathrm{h}$ if $b=0$ and $c<0$.

It is clear that Figure f leads to Figure 16, Figure g leads to Figure 7 and Figure $\mathrm{h}$ leads to Figure 10 or 11.

4.3. The (c.2.2) case. We have $\left(y^{2}+n x+p\right) d x+(a y+b x+c) d y=0$ with either $n \neq 0$ or if $n=0$, then $p>0$.

$V=\left(V_{1}, V_{2}\right)=\left(Y^{2}+n Z+p Z^{2}+a Y^{2} Z+b Y Z+c Y Z^{2} ; a Y Z^{2}+b Z^{2}+c Z^{3}\right)$.

Blowing up $(0,0)$ by $f(y, z)=(y, y z)$ we get $U=\left(U_{1}, U_{2}\right)$ given by

$$
\begin{aligned}
& U_{1}=y^{2}+n y z+p y^{2} z^{2}+a y^{3} z+b y^{2} z+c y^{3} z^{2} \\
& U_{2}=-y z-n z^{2}-p y z^{3} .
\end{aligned}
$$

If $n=0$ we can divide by $y$ and see that $U / y$ has $(0,0)$ as its only singularity, with derivative $\left(\begin{array}{ll}1 & * \\ 0 & -1\end{array}\right)$. 

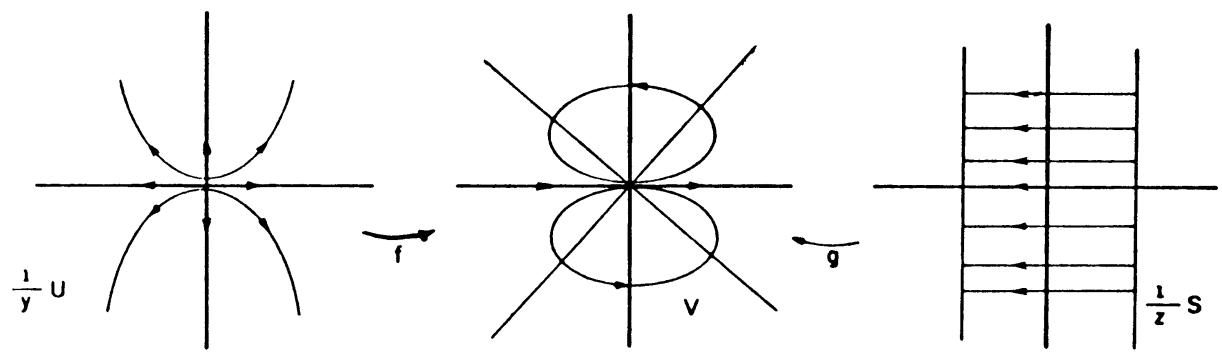

FIGURE i
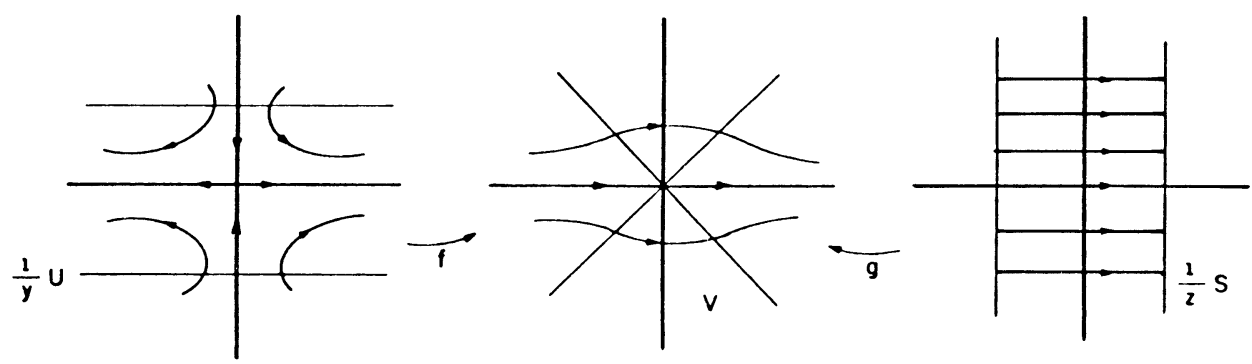

FiguRE j

If $n \neq 0, S=g^{*} V$ is nonsingular at $(0,0)$. If $n=0, S=z S^{\prime}$ and $S^{\prime}(0,0) \neq 0$, so it is clear, that if $n=0$ we have Figure $i$.

It remains to blow up the origin for the vector field $U$ in the case $n \neq 0$ since this is its only singularity.

Let $\bar{U}=\left(\bar{U}_{1}, \bar{U}_{2}\right)$ be the vector field obtained by $U$ using the directional blowing up $f(y, z)=(y, y z)$.

$$
\begin{aligned}
& \bar{U}_{1}=y^{2}+n y^{2} z+p y^{4} z^{2}+a y^{4} z+b y^{3} z+c y^{5} z^{2} \\
& \bar{U}_{2}=-2 y z-2 n y z^{2}-2 p y^{3} z^{3}-a y^{3} z^{2}-b y^{2} z^{2}-c y^{4} z^{3}
\end{aligned}
$$

$\bar{U} / y$ has singularities at $(0,0)$ and at $(0,-1 / n)$ with derivatives, respectively, equal to

Since

$$
\left(\begin{array}{cc}
1 & 0 \\
0 & -2
\end{array}\right) \text { and }\left(\begin{array}{cc}
0 & 0 \\
-b / n^{2} & 2
\end{array}\right) \text {. }
$$

$$
\frac{1}{y} \bar{U}_{1}\left(y,-\frac{1}{n}\right)=-\frac{b}{n} y^{2}-\left(\frac{a}{n}-\frac{p}{n^{2}}\right) y^{3}+\frac{c}{n^{2}} y^{4}
$$

and $b \neq 0$ (nonintersection condition) we have a saddle-node at $(0,-1 / n)$.

Let $\tilde{U}=\left(\tilde{U}_{1}, \tilde{U}_{2}\right)$ be the vector field obtained by $U$ using the directional blowing $\operatorname{up} g(y, z)=(y z, z)$.

$$
\begin{aligned}
& \tilde{U}_{1}=2 n y z+2 y^{2} z+2 p y^{2} z^{3}+a y^{3} z^{3}+b y^{2} z^{2}+c y^{3} z^{4} \\
& \tilde{U}_{2}=-n z^{2}-y z^{2}-p y z^{4}
\end{aligned}
$$

It is clear that the origin is a saddle point for $\tilde{U} / z$. Its derivative there is $\left(\begin{array}{cc}2 n & 0 \\ 0 & -n\end{array}\right)$. From $\bar{U}$ and $\tilde{U}$ we get $U$ (Figure j) and from $U$ and $S$ we get $V$ (Figure k). 

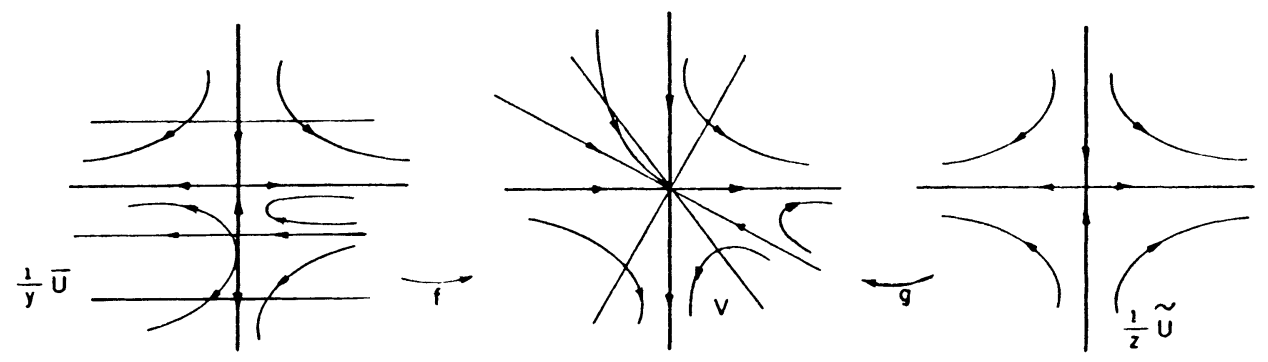

FIGURE k

It is clear that Figure $\mathrm{i}$ leads to Figure 7 and Figure $\mathrm{k}$ leads to Figure 19.

4.4. The (c3) case. Our equation is $(a x+b y+c) d x+\left(y^{2}+e x\right) d y=0$ with $a \neq 0$.

The associated vector field $V=\left(V_{1}, V_{2}\right)$ is given by

$$
V_{1}=a Z+b Y Z+c Z^{2}+Y\left(Y^{2}+e Z\right) ; \quad V_{2}=Z\left(Y^{2}+e Z\right) .
$$

Blowing up $(0,0)$ by $f(y, z)=(y, y z)$ we get $U=\left(U_{1}, U_{2}\right)$ given by

$$
U_{1}=a y z+(b+e) y^{2} z+c y^{2} z^{2}+y^{3} ; \quad U_{2}=-a z^{2}-b y z^{2}-c y z^{3} .
$$

$U$ has $(0,0)$ as its only singularity, with derivative zero.

We will blow up $(0,0)$ for the vector field $U$ getting $\bar{U}=\left(\bar{U}_{1}, \bar{U}_{2}\right)$ (when we use $f$ ) and $\tilde{U}=\left(\tilde{U}_{1}, \tilde{U}_{2}\right)$ (when we use $g$ ). We have

$$
\begin{aligned}
& \bar{U}_{1}=a y^{2} z+(b+e) y^{3} z+c y^{4} z^{4}+y^{3} \\
& \bar{U}_{2}=-2 a y z^{2}-(2 b+e) y^{2} z^{2}-2 c y^{3} z^{3}-y^{2} z \\
& \tilde{U}_{1}=2 a y z+(2 b+e) y^{2} z^{2}+2 c y^{2} z^{3}+y^{3} z^{2} \\
& \tilde{U}_{2}=-a z^{2}-b y z^{3}-c y z^{4} .
\end{aligned}
$$

$\bar{U} / y$ has $(0,0)$ as its only singularity, but it is a degenerate one, which will require another blow up.

$\tilde{U} / z$ has $(0,0)$ as its only singularity with derivative $\left(\begin{array}{cc}2 a & 0 \\ 0 & -a\end{array}\right)$.

Let $T$ and $\tilde{T}$ be the results of blowing up the origin for the vector field $\bar{U} / y$ using respectively the maps $f$ and $g$.

If $T=\left(T_{1}, T_{2}\right)$ and $\tilde{T}=\left(\tilde{T}_{1}, \tilde{T}_{2}\right)$ we have

$$
\begin{aligned}
& T_{1}=a y^{2} z+(b+e) y^{3} z+y^{2}+c y^{5} z^{2}, \\
& T_{2}=3 a y z^{2}-(3 b+2 e) y^{2} z^{2}-3 c y^{3} z^{3}-2 y z, \\
& \tilde{T}_{1}=3 a y z+(3 b+2 e) y^{2} z^{2}+2 y^{2} z+3 c y^{3} z^{4}, \\
& \tilde{T}_{2}=-2 a z^{2}-(2 b+e) y z^{3}-2 c y^{2} z^{5}-y z^{2} .
\end{aligned}
$$

$\tilde{T} / y$ has singularities at $(0,0)$ and $(0,-2 / 3 a)$ with derivatives respectively equal to

$$
\left(\begin{array}{cc}
1 & 0 \\
0 & -2
\end{array}\right) \text { and }\left(\begin{array}{cc}
1 / 3 & 0 \\
-4 b / 3 a^{2} & 2
\end{array}\right) \text {. }
$$

$\tilde{T} / z$ has singularities at $(0,0)$ and at $(-3 a / 2,0)$ and as we remarked before we only need to study $(0,0)$, where $\tilde{T} / z$ has derivative $\left(\begin{array}{cc}3 a & 0 \\ 0 & -2 a\end{array}\right)$. 
858

M. I. T. CAMACHO AND C. F. B. PALMEIRA

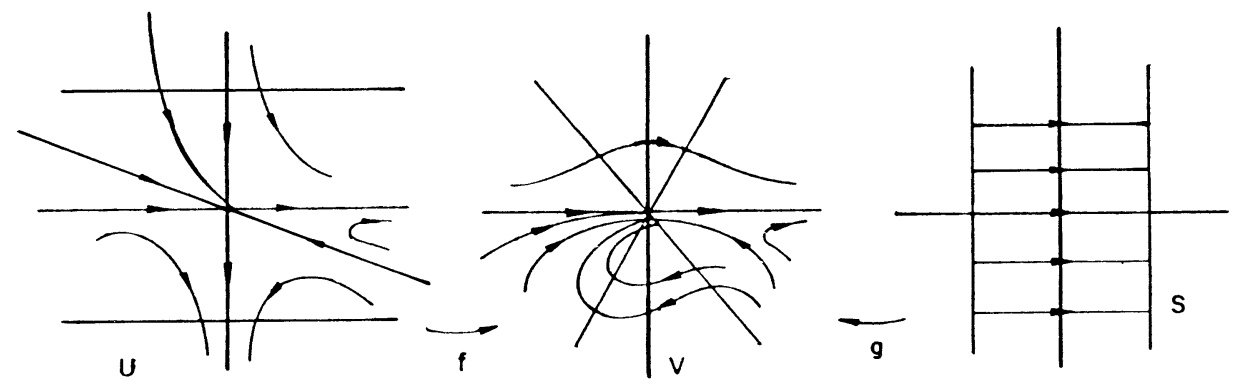

FIGURE 1
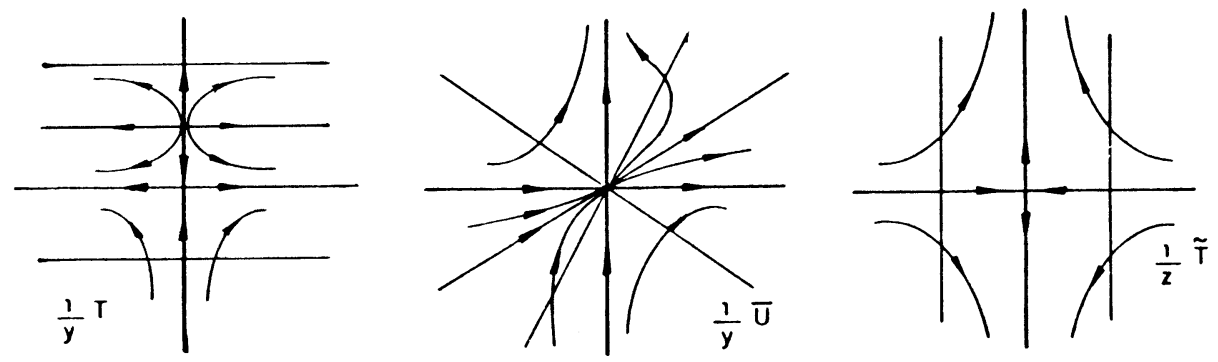

FIGURE m
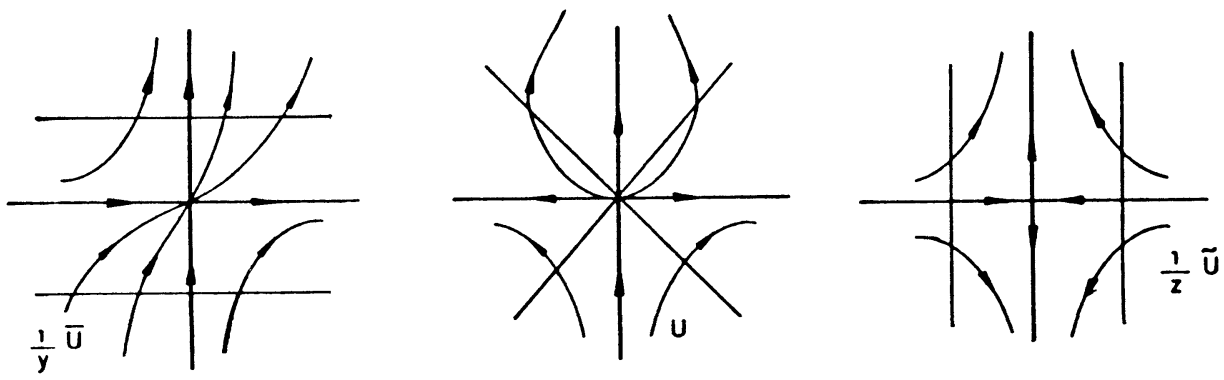

FIGURE $\mathrm{n}$
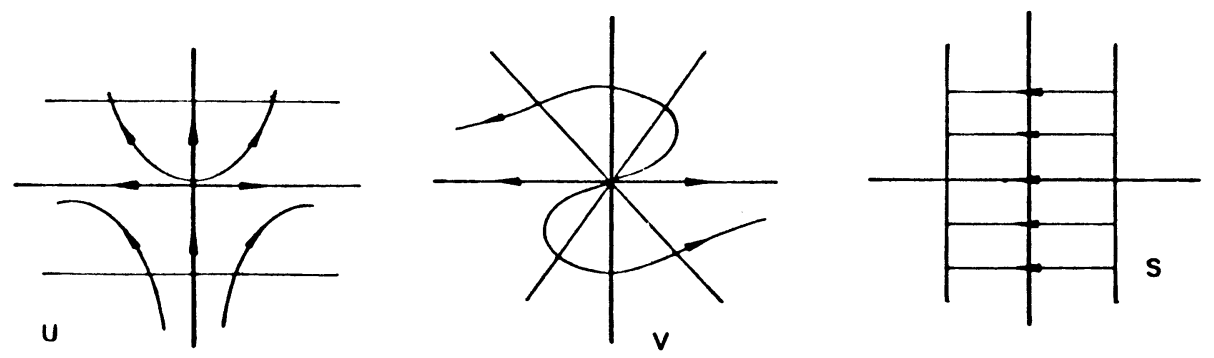

Figure o 
From $T / y$ and $\tilde{T} / z$ we get $\bar{U} / y$ (Figure 1 ); from $\bar{U} / y$ and $\tilde{U} / z$ we get $U$ (Figure $\mathrm{m}$ ). From $U$ and $S$ we get $V$ (Figure n) $\left(S=g^{*} V\right.$ is again nonsingular at $\left.(0,0)\right)$.

It is easy to see that Figure $\mathrm{n}$ leads to Figure 7.

\section{REFERENCES}

[A] V. Arnold, Chapitres supplémentaires de la théorie des équations différentielles ordinaires, Éditions de Moscou.

[C.T.] C. Chicone and Tian Jinghuang, On general properties of quadratic systems, Amer. Math. Monthly 89 (1982), 167-178.

[D] F. Dumortier, Singularities of vector fields, Monograf. Mat. 32, IMPA, 1978.

[G.S.L.] A. Gasull, Sheng Li-Ren and J. Llibre, preprint Univ. Autonoma Barcelona.

[Ma] L. Markus, Topological types of polymomial differential equations, Trans. Amer. Math. Soc. 171 (1972), 157-178.

[Mu] M. P. Muller, Quelques propriétés des feuilletages polynomiaux du plan, Bol. Soc. Math. Mexicana 21 (1976), 6-14.

[P] C. F. B. Palmeira, Open manifolds foliated by planes, Ann. of Math. 107 (1978), 109-131.

[S] Sheng Li-Ren, Systèmes quadratiques et composantes de Reeb, Publication de l'IRMA, Univ. de Strasbourg, 1984, 226/p-132.

[S.S.] S. Schecter and M. Singer, Planar polymomial foliations, Proc. Amer. Math. Soc. 79 (1980), 649-656.

[S.S.2] _ Singular points of planar vector fields, Lecture Notes in Math., vol. 819, Springer-Verlag, Berlin and New York.

[T] F. Takens, Singularities of vector fields, Inst. Hautes Études Sci. Publ. Math. 43 (1974), 47-100.

Instituto de Matemática, Universidade Federal do Rio de Janeiro, IlHa do FUNDÃO, RIO DE JANEIRO RJ 21910, BRASIL

Departamento de matemática, Pontifícia Universidade, Católica do Rio DE JANEIRO, RIO DE JANEIRO RJ 22453, BRASIL 